\title{
How deep is the inflammation in chronic rhinosinusitis? Sinus wall thickness and blood eosinophilia*
}

\author{
Kuei-You Lin ${ }^{1,2}$, Pey-Yu Chen' ${ }^{1}$ Te-Huei Yeh'1 \\ ' Department of Otolaryngology, National Taiwan University Hospital, Taipei, Taiwan \\ 2 Department of Otolaryngology, Shin Kong Wu Ho-Su Memorial Hospital, Taipei, Taiwan
}

Rhinology 56: 46-53, 2018

https://doi.org/10.4193/Rhino15.250

*Received for publication:

August 18, 2015

Accepted: December 26, 2016

\begin{abstract}
Various factors have been proposed to be related to refractory chronic rhinosinusitis (CRS). Treatment for refractory CRS is challenging for ear, nose, and throat (ENT) surgeons. The aim of the study was to determine the clinical features associated with the severity of CRS that may necessitate revision surgery by eliminating the bias of the surgeon's technique using standardizing surgical procedures. Sinus wall thickness and blood eosinophilia, which may represent the depth of inflammation in CRS, are associated with the need for revision surgery. We found that, when the thickness of the postero-lateral maxillary sinus wall is more than 3.03 $\mathrm{mm}$, there is an increased probability for a need for revision surgery. CRS patients with thickened sinus walls were found to have poorer outcomes. Further research is needed in order to justify this type of surgical procedure for CRS.
\end{abstract}

Key words: refractory, chronic rhinosinusitis, inflammation, osteitis, eosinophilia

\section{Introduction}

The sinonasal organ plays an important role in the human respiratory system, as this organ consistently encounters external irritants and is therefore one of the most frequently inflamed sites in the human body ${ }^{(1)}$. Inflammation may begin as an infectious process (acute rhinosinusitis) and, if the symptoms persist without resolution, it can lead to inflammatory consequences (chronic rhinosinusitis; CRS) ${ }^{(2)}$. CRS is one of the most prevalent chronic diseases in modern society and is defined as the presence of more than one nasal symptom (mucopurulent drainage, nasal congestion, facial pain-pressure-fullness, and decreased sense of smell) and a documentation of inflammation for more than 12 weeks ${ }^{(3)}$. It is a heterogeneous, multifactorial disease with multiple distinct factors, including genetic, infectious, immune, anatomic, allergic, and inflammatory components ${ }^{(2)}$. The goal of CRS therapy is maximal medical treatment including oral and topical antibiotics, nasal steroids, systemic steroids, antihistamines, and saline irrigations, etc. Functional endoscopic sinus surgery (FESS) is indicated if medical therapy fails ${ }^{(4)}$.

The safety and efficacy of FESS for CRS has been strongly supported by meta-analyses from both large outcome studies, as well as cohort studies. Improvement in both disease-specific and generic quality-of-life, as well as objective measures, have been demonstrated for the efficacy of FESS; however, across long-term follow up, there is a $10-20 \%$ revision rate, which is considered to be refractory CRS ${ }^{(5)}$. Refractory CRS is defined by failure to stabilize after surgery and treatment with antibiotics, saline rinses, and topical steroid, and has become a significant issue for ENT surgeons ${ }^{(6)}$. Predicting surgical outcome is crucial for evaluating the severity of CRS preoperatively, and the severity of CRS is usually defined by several factors. Temporally, the duration and frequency of symptoms and signs of CRS patients cannot be precisely correlated. Spatially, the Lund-Mackay (L-M) score, which is based on CT images, is the most frequently used method to evaluate the severity of CRS; nevertheless, the L-M score represents only a snap shot of the condition ${ }^{(7)}$. A swift change in mucosal swelling is frequently observed during the subacute stage of sinusitis. Other parameters should be considered in order to define the severity of CRS and more accurately predict its prognosis.

Various factors are related to refractory CRS, including mucociliary dysfunction, the presence of mucosal biofilm, peripheral eosinophil count, mucosal eosinophilia, acute post-operative infection, ASA triad, cystic fibrosis, osteitis, hyperreactive airway, 
inhaled allergen, and experience of the performing surgeon ${ }^{(8-12)}$. It is important to find a simple way to evaluate the severity of CRS in order to identify an accurate prognosis for patients and determine which patients may need long-term medical treatment. The aim of the study was to determine the clinical features related to the severity of CRS that would necessitate revision surgery, by carefully eliminating surgeon bias using standardizing surgical procedures.

\section{Materials and methods}

Standardization of surgical procedure and treatment protocol

The first consideration of this study was to select appropriate patients in order to exclude the congenitally influential factor of heterogenecity of CRS. The next consideration was to standardize the preoperative treatment, surgical procedure, and postoperative follow-up protocol. Over the past 10 years, we have developed a standardized surgical procedure and treatment protocol. Preoperatively, the referral doctor administers optimal medical treatment to the patient; if that does not occur, our clinics will administer the treatment. If treatment fails, surgery is suggested. Preoperative medication is not given for at least 2 weeks, if acute exacerbation was not noted before surgery. Some patients are given a loose schedule of intranasal steroid spray but oral antibiotics are not given regularly. Other information is also collected during the preoperative visit: age, sex, asthma, nasal polyps, allergic rhinitis, obstructive sleep apnea, diabetes, smoking status, gastroesophageal reflux disease, prior FESS history, and Samter's triad. For the surgical procedure, the objects of FESS include several folds: to clear out the occluded ostium by correcting the anatomical flaw of bottle neck of draining pathway for diseased sinus, to decrease inflammatory load by removing developed polyps or swollen mucosa which was filled with inflammatory milieu, and to clean out entrapped discharge from deep seated recess which contained inflammation inducing materials. Based on the principles described above, we developed 8 complete steps to perform standard FESS:

1. Middle turbinate trimming: this procedure is used instead of medial fracture in order to expose the posterior margin of uncinate process and hiatus semilunaris.

2. Uncinectomy: the first step of ethmoidectomy is to properly remove the uncinated process. It can be antegrade, with a sickle knife, or retrograde, by using back biting forceps, until the superiorly agger nasi cell and frontal recess and inferiorly the natural ostium of maxillary sinus are identified.

3. Enlargement the natural ostium of maxillary sinus: by removing the mucosa of posterior fontanell, the accessory ostium can be identified during this step. Pathologic tissue in the maxillary sinus is removed, and irrigation by normal saline is frequently applied.
4. Removal of the bony wall of the ethmoid bulla: identifying the basal lamella of the middle turbinate. The retrobullar recess and suprabullar cells are exposed. It is important to expose and identify lamina papyracea in order to delineate the lateral margin of the ethmoid cavity.

5. Removal of the basal lamella at the medial inferior site: in order to enter posterior ethmoid sinus space safely, the middle turbinate insertion is not destabilized. When entering posterior ethmoid space, the superior turbinate and skull base are identified by carefully removing the ethmoid bony septum of the posterior ethmoid sinuses.

6. Opening of the sphenoid sinus, if necessary: The sphenoid sinus is not frequently involved in CRS. If necessary, remove the inferior third of superior turbinate in order to easily find the natural ostium of sphenoid sinus. Irrigation or removing polypoid mucosa surround ostium is sufficient for most patients.

7. After identifying the skull base at posterior ethmoid roof, anterior removal of the bony septum along the skull base to reach the bony indentation of the anterior ethmoid artery (AEA), which is the most important landmark to manage frontal recess. The AEA is a landmark ("Nike" logo-shaped curve) and may be buried inside a bony canal in patients with well-pneumatized anterior ethmoid sinuses; it could be mesenteric. This space is complicated by various possible suprabullar or frontobullar cells and is a challenging step for beginners.

8. Cleaning frontal sinus draining route: Using the 45-degree endoscope, mark the anatomic landmark of AEA posteriorly and agger nasi or frontoethmoid cells anteriorly; the frontal sinus-draining route is frequently buried in the complicated anterior ethmoid cells system. There may be anteriorly, posteriorly, medially, and laterally-located ethmoid air cells; it is important to identify the boundary of ethmoid cavity laterally to the lamina papyracea, medially to the middle turbinate concha; and superiorly along the skull base. The frontal sinus will be safely opened.

Postoperative care includes regularly follow-up for at least 6 months using optimal antibiotics treatment, including low-dose macrolide for 2 months and nasal steroids, as well as routine saline nasal douching plus adjuvant of gentamicin for at least 3 months.

\section{Patient selection}

We reviewed the medical records of 243 patients who received bilateral FESS in our department by the same senior surgeon of a tertiary referral hospital from September 2010 to August 2011. Computed tomography (CT) of paranasal sinuses was performed in all patients at most 2 months prior to surgery. Other preoperative evaluations included hematologic examination the 


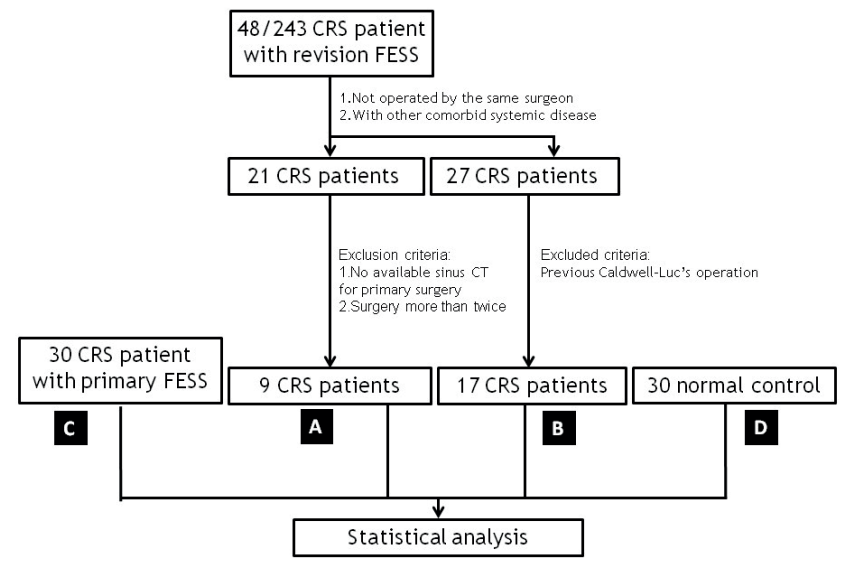

Figure 1. Flow chart of patient selection.

day before surgery. Patients with known systemic diseases or malignancies were excluded, such as diabetes mellitus, asthma, or other immunocompromised diseases. 48 CRS patients who received revised sinus surgery during this period were recruited; 21 of these patients received surgery by the same surgeon and the other 27 patients received surgery from different surgeons. Among the 21 patients, patients with no previous sinus CT scan available for comparison was excluded. Among 27 patients, we excluded the patients who received previous Caldwell-Luc procedures. 9 patients were assigned to group A (primary and revised surgery by the same senior surgeon), 17 patients were assigned to group $B$ (previously operated on by other surgeons), and 30 control patients were assigned to Group $C$ who received primary FESS surgery during the same period and were followed-up for at least 3 years without revision surgery. Group $D$ included 30 control patients with head and neck CT scan from parotid surgery without notified sinonasal problems. Hematologic examination was evaluated for Group D patients one day prior to surgery. The flow chart of patient selection is shown in Figure 1. The clinical information including result of bacterial culture, pathology to evaluate eosinophilic rhinosinusitis, with or without asthma and ImmnoCAP Specific IgE blood test were also collected for further analysis.

\section{Radiographic evaluation}

The extent of paranasal sinus mucosal disease was evaluated by using the L-M staging system ${ }^{(7)}$. Sinus wall thickness was measured in coronal view of the sinus CT. The posterolateral wall of maxillary sinus, para-crista galli level (around the lateral lamella) of the ethmoid sinus, and the anterior clinoid level of sphenoid sinus were measured as the representative thickness of each sinus wall (Figure 2). All CT studies were performed by 64 multidetector-row CTs (Lightspeed VCT, GE Medical Systems). Contiguous axial $1.2 \mathrm{~mm}$ thick slices were obtained through the maxillofacial bones, and images were reconstructed with soft tissue and bone reconstruction algorithms and $3.0 \mathrm{~mm}$ thick

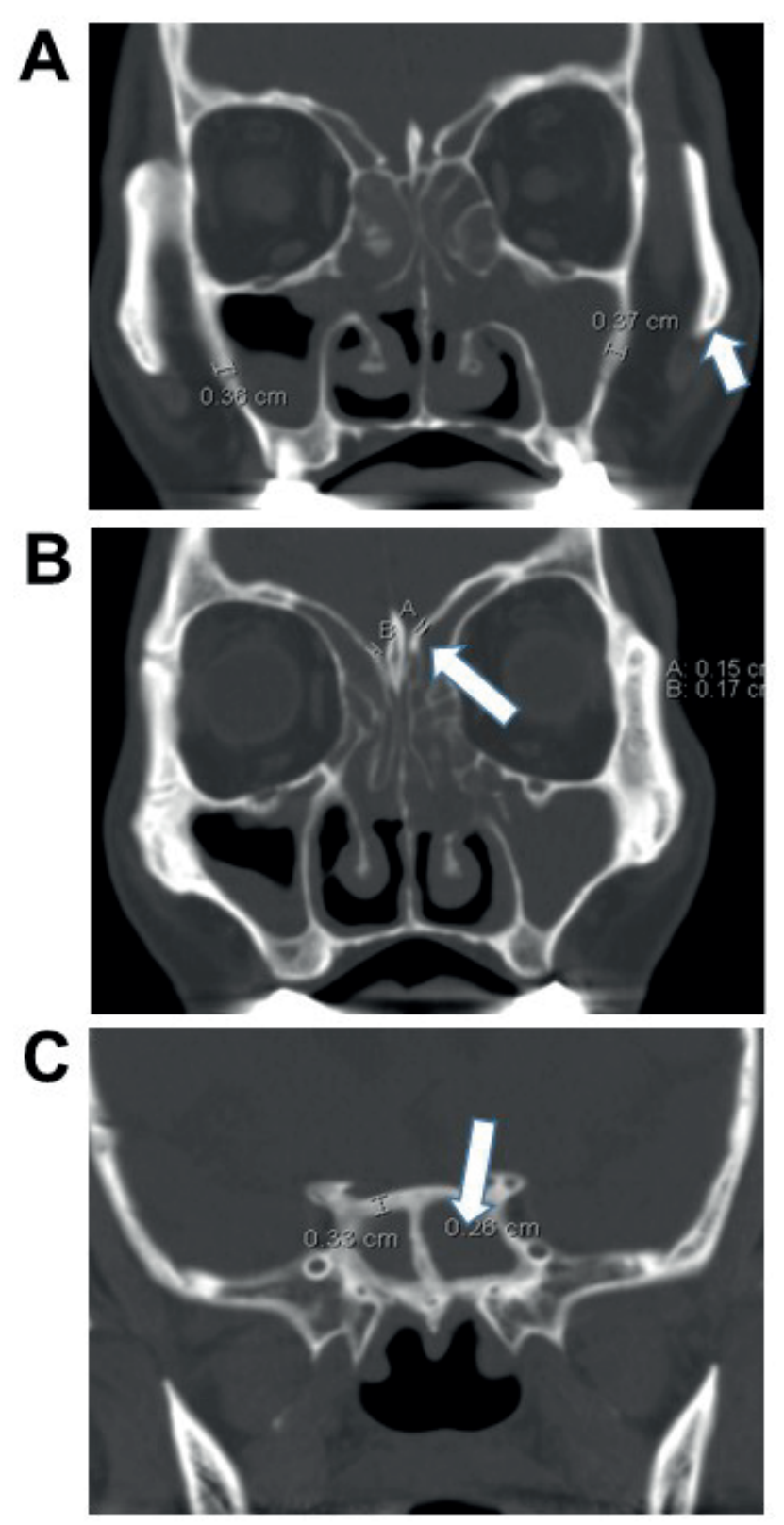

Figure 2. Representative sinus CT scan to measure sinus wall thickness. A 61-year-old male patient with recurrent CRS and status post 2 times of FESS; the figure shows the pre-operative sinus CT image of first FESS. A) Maxillary sinus wall thickness is measured at the posterolateral region. The first cut shown when the zygoma (white arrow) is separated from maxillary sinus wall, measuring the thickest part. B) Ethmoid sinus wall thickness is measured at the para-crista galli region around lateral lamella, the thickest part (white arrow). C) The sphenoid sinus wall thickness is measured at the first cut showing the anterior clinoid, the thickest part (white arrow).

coronal and sagittal reformatted images were obtained per our institutional protocol. All CT measurements were made on bone algorithm reconstructed and bone windowed images (W:2000, L:500) using an independent workstation. The measurement 
Table 1. Patients' demographic data.

\begin{tabular}{|c|c|c|c|c|c|c|}
\hline Group & $\mathbf{N}$ & Age & Gender* & Bacteriology $y^{*}$ & Eosinophilic rhinosinusitis ${ }^{s}$ & Aspirin intolerance or Asthma \\
\hline A & 9 & $49.1 \pm 15.1$ & $8 \mathrm{M} 1 \mathrm{~F}(1)^{8}$ & 3 & 1 & 1 \\
\hline B & 17 & $47.8 \pm 15.6$ & $11 \mathrm{M} 6 \mathrm{~F}(3)$ & 3 & 1 & 1 \\
\hline C & 30 & $41.9 \pm 11.3$ & $23 M 7 F(4)$ & 2 & 1 & 0 \\
\hline D & 30 & $44.7 \pm 13.6$ & $10 \mathrm{M} 20 \mathrm{~F}$ & NA & NA & NA \\
\hline
\end{tabular}

Group A: revision group (same surgeon); Group B: revision group (different surgeons); Group C: primary FESS group; Group D: normal control group. * $p<0.05$, (Kruskal-Wallis test); *: other than normal flora, there are Staphylococcus aureus $(n=5)$; MRSA $(n=1)$; Citrobacter kerosi $(n=1)$; Hemophilus

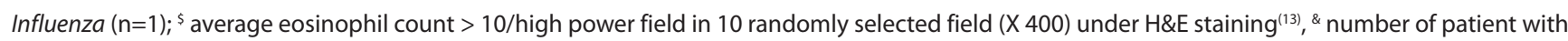
positive ImmnoCAP test.

Table 2. Hematologic examination result in different groups.

\begin{tabular}{|c|c|c|c|c|c|}
\hline \multirow[t]{2}{*}{ Group } & \multicolumn{5}{|c|}{ Hematologic examination } \\
\hline & Hemoglobin & WBC count $(k / \mu l)$ & Eos. (\%) & $\begin{array}{l}\text { Peripheral Eos. Count } \\
(\mathrm{k} / \mu \mathrm{l})\end{array}$ & Sugar (g/dL) \\
\hline A & $14.58 \pm 0.67$ & $6333.3 \pm 1494.5$ & $4.48 \pm 3.82$ & $303.09 \pm 297.48^{*}$ & $91.2 \pm 8.3$ \\
\hline B & $14.34 \pm 1.29$ & $6119.4 \pm 1781.6$ & $2.91 \pm 2.03$ & $181.45 \pm 179.31$ & $93.1 \pm 14.3$ \\
\hline C & $14.29 \pm 1.53$ & $6994.3 \pm 1637.0$ & $3.66 \pm 3.03$ & $249.48 \pm 240.49$ & $90.1 \pm 9.2$ \\
\hline D & $14.00 \pm 1.23$ & $6146.7 \pm 1598.4$ & $3.50 \pm 2.51$ & $164.29 \pm 204.15$ & $96.4 \pm 21.6$ \\
\hline
\end{tabular}

Group A: revision group (same surgeon); Group B: revision group (different surgeons); Group C: primary FESS group; Group D: normal control group. * $\mathrm{p}<0.05$, compared with group $\mathrm{D}$.

were repeated by two independent otolaryngologist. In detail, maxillary sinus wall thickness is measured at the posterolateral region where the first cut shown when the zygoma is separated from maxillary sinus wall, measuring the thickest part. The ethmoid sinus wall thickness is measured at the para-crista galli region around lateral lamella, also the thickest part. The sphenoid sinus wall thickness is measured at the first cut showing the anterior clinoid, the thickest part. At lateral sphenoid wall, the carotid canal and optic nerve may introduce measurement confusion, and it's the reason why we choose anterior clinoid level instead of lateral sphenoid wall for wall thickness measurement where more constant sinus wall thickness could be measured. Frontal sinus wall was excluded due to its high variation of pneumatization in normal population.

\section{Statistics analyses}

We assessed gender, age, hemoglobin $(\mathrm{Hb})$ levels, white blood cell (WBC) count, eosinophilic count, L-M score, bony wall thickness of maxillary/ethmoid/sphenoid sinus, and mean recurrence time of CRS. Comparisons between groups were performed by analysis of variance (ANOVA) tests for normal distributed variables and Kruskal-Wallis test for non-parametric variables, as required. Chi-square tests for categorical variables. Multiple logistic regression analysis was performed between group $A$ and $C$ against various parameters including age, gender and maxillary sinus wall thickness, L-M score, $\mathrm{Hb}$ and eosinophil count. Receiver operating characteristic (ROC) curve were constructed to define cut-off value for potential refractory CRS. Linear regression was used to determine the relationship between scalar variables. All statistical analyses were performed using SPSS software. $P<0.05$ was considered to be statistically significant.

\section{Results}

\section{Patient demographics}

Group A included 8 males and 1 female (mean age: $49.1 \pm 15.1$ years), group $B$ included 11 males and 6 females (mean age: $47.8 \pm 15.6$ years), group $C$ included 23 males and 7 females (mean age: $41.9 \pm 11.3$ years), and group $D$ included 10 males and 20 females (mean age: $50 \pm 14.9$ years). There was no significantly difference across age (unpaired t-test; $p>0.05$ ). The gender distribution was significantly different with obvious male preponderance in Group A, B, and C (Kruskal-Wallis test; $p<0.05$; Table 1). The information including bacteriology, eosinophilic rhinosinusitis, asthma or ImmnoCAP Specific IgE blood test were shown in Table 1. The bacteriological data indicated Staphylococcus aureus was the major bacterial specie found. The main 
Table 3. L-M score and sinus wall thickness result in different groups.

\begin{tabular}{ccccc} 
Group & L-M score & \multicolumn{3}{c}{ sinus wall thickness (mm) } \\
& & maxillary & ethmoid & sphenoid \\
\hline A & $16.7 \pm 8.0$ & $4.25 \pm 1.66$ & $1.83 \pm 0.37$ & $1.89 \pm 0.66$ \\
\hline B & $12.9 \pm 6.6^{5}$ & $2.43 \pm 0.83$ & $1.49 \pm 0.28$ & $1.49 \pm 0.59$ \\
\hline C & $12.9 \pm 5.1$ & $2.06 \pm 0.49^{\natural}$, & $1.46 \pm 0.24^{\natural}$ & $1.34 \pm 0.28^{\natural}$ \\
\hline D & $0.23 \pm 0.43$ & $1.97 \pm 0.42^{1, \#}$ & $1.44 \pm 0.33^{\natural}$ & $1.39 \pm 0.34^{\natural}$
\end{tabular}

Group A: revision group (same surgeon); Group B: revision group (different surgeons); Group C: primary FESS group; Group D: normal control group. ": $p<0.05$, compared with group $A ;$;: $p<0.05$, compared with group $B$; ${ }^{\text {s: }}$ with primary $C T ; n=8$.
Table 4. Multiple logistic regression between group $\mathrm{A}$ and $\mathrm{C}$.

\begin{tabular}{|lccc|}
\hline & P value & Odds ratio & $95 \% \mathrm{Cl}$ \\
\hline Age & 0.648 & 1.039 & $0.881 \sim 1.226$ \\
\hline Gender & 0.711 & 0.001 & $0.026 \sim 8.020$ \\
\hline Maxillary thickness & 0.037 & 19.442 & $1.192 \sim 317.181$ \\
\hline L-M score & 0.958 & 0.994 & $0.800 \sim 1.235$ \\
\hline Hb. & 0.648 & 1.400 & $0.330 \sim 5.951$ \\
\hline Eosinophil count & 0.827 & 0.999 & $0.989 \sim 1.009$ \\
\hline
\end{tabular}

Group A: revision group (same surgeon)

Group C: primary FESS group infiltrative inflammatory cells according to pathologic slides were lymphoplasma cells and occasionally, neutrophil infiltration. Eosinophilic rhinosinusitis was defined as average eosinophil count $>10 /$ high power field in 10 randomly selected field ( $x 400)$ under H\&E staining from our previous publication ${ }^{(13)}$. Aspirin intolerance or bronchial asthma were very scarce (only one case in group $A$ and $C$, respectively).

\section{Hematologic examination}

There was no statistically significant difference in hematologic examination results between groups (unpaired t-test; $p>0.05$ ). The absolute peripheral eosinophil counts in group $\mathrm{A}$ were significantly higher than in group D (Table 2).

\section{L-M Score and Sinus wall thickness}

The average L-M score of group A was $16.7 \pm 8.0$ and of group C was 12.9 \pm 5.1 ; there was no significant difference between these groups (unpaired t-test; $p>0.05$, Table 3 ).

The assessments of sinus wall thickness between the independent otolaryngologists were very close. The average intraclass correlation coefficient between two assessors was 0.833 (95\% confidence interval: $0.782-0.872$ ). In terms of individual sinuses, the closest interrater agreement was found for sphenoid sinuses $(0.821,0.932)$, followed by the ethmoid sinuses $(0.631,0.851)$ and the maxillary sinuses $(0.576,0.825)$. In group $A$, the mean sinus wall thickness of the maxillary, ethmoid, and sphenoid sinuses were $4.25 \pm 1.66 \mathrm{~mm}, 1.83 \pm 0.37 \mathrm{~mm}$, and $1.89 \pm 0.66 \mathrm{~mm}$, respectively. Group B had a mean thickness of $2.43 \mathrm{~mm} \pm 0.83$ $\mathrm{mm}, 1.49 \pm 0.28 \mathrm{~mm}$, and $1.49 \pm 0.59 \mathrm{~mm}$ in the maxillary, ethmoid, and sphenoid sinuses. In group C, the sinus wall thickness was as follows: maxillary sinus: $2.06 \pm 0.49 \mathrm{~mm}$; ethmoid sinus: $1.46 \pm 0.24 \mathrm{~mm}$; sphenoid sinus: $1.34 \pm 0.28 \mathrm{~mm}$. In group $D$, the measured sinus wall mean thickness of the maxillary, ethmoid, and sphenoid sinuses were $1.97 \pm 0.42 \mathrm{~mm}, 1.44 \pm 0.33$ $\mathrm{mm}$, and $1.39 \pm 0.34 \mathrm{~mm}$, respectively.
The sinus wall thickness of group $A$ was significantly higher than groups $C$ and $D$; in group $B$, only the maxillary sinus wall thickness was significantly different compared to group $D$ (unpaired t-test; $\mathrm{p}<0.05$; Table 3). Multiple logistic regression analysis was performed between group $A$ and $C$ against various parameters including age, gender and maxillary sinus wall thickness, L-M score, $\mathrm{Hb}$ and eosinophil count. Eventually, maxillary sinus wall thickness was an independent significant factor noted $(p=0.037$; Table 4).

\section{Mean recurrence time}

Average recurrence time of group A was $28.7 \pm 13.9$ months. There was no significant correlation between the mean recurrence time and pre-operative L-M score (Figure 3 ) or sinus wall thickness (linear regression, $p>0.05$, Figure 4).

The cut-off values of sinus wall thickness for the prediction of recalcitrant CRS

The cut-off value of sinus wall thickness in prediction of refractory CRS who needs revision surgery differed across sinuses. The sensitivity and specificity for prediction also varied. Using $3.03 \mathrm{~mm}$ as a cut-off value for the maxillary sinus, the sensitivity was $88.9 \%$ and the specificity was $90 \%$. Using $1.63 \mathrm{~mm}$ as cut-off value for ethmoid sinus, the sensitivity and specificity were $77.8 \%$ and $80.0 \%$, respectively. Using $1.75 \mathrm{~mm}$ as a cut-off value for the sphenoid sinus, the sensitivity was $44.4 \%$ and the specificity was $80.0 \%$.

Different sinus wall thicknesses showed areas under ROC curve (AUC) of 0.94, 0.72, and 0.63, for maxillary, ethmoid and sphenoid sinuses, respectively. The AUC of maxillary sinus wall thickness reached statistical significance (ROC curve; $p<0.05$, Figure 5).

\section{Discussion}

The success of FESS for CRS is still variable, ranging from $76 \%$ 


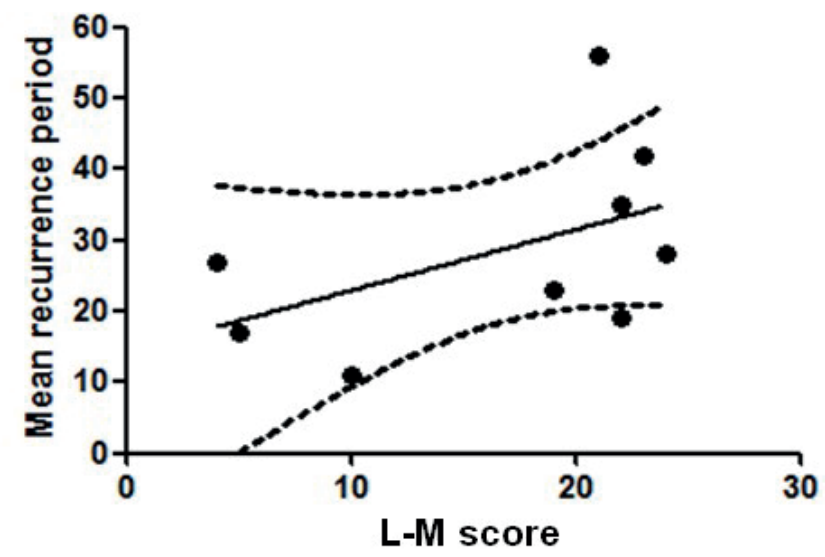

Figure 3. Linear regression between the mean recurrence period and L-M score in group A showed no significant relationship ( $p>0.05$ ).

to $98 \%{ }^{(4)}$. Although the exact predictive factors are still controversial, several risk factors, such as nasal polyps, allergic rhinitis, aspirin intolerance, and bacterial resistance, can result in unfavorable treatment outcomes for CRS patients, In this study, we found that the presence of thickened maxillary sinus walls of more than $3.03 \mathrm{~mm}$, as well as increased peripheral blood eosinophil count, are good predictors of unfavorable outcomes from FESS. In addition to the classical L-M scoring system, a 2-dimensional parameter, we suggest that these 2 factors may represent another 3-dimensional parameter that may indicate the depth of inflammation in CRS in order to evaluate its severity.

The sinonasal organ is an expanding air-filled space that grows in a random pattern proven by using a simple computerized equation ${ }^{(14)}$. The sinus pneumatization process may be considered as using limited material ballooning to occupy the space among the eyeballs, brain, and mouth. This expanding process creates one frontal, maxillary, and sphenoid sinus cell on each side, and more importantly, the complexity of the ethmoid cell system. The bony sinus wall, which confines this sinonasal cavity, represents the boundary and most peripheral lining of this organ. If it is involved in the inflammatory process, it might be considered as one of the dimensions of the depth of disease extent, indicating a more severe form of sinonasal disease. One study showed that CRS patients had smaller maxillary sinuses than normal controls. These authors proposed that the increased bone thickness in the maxillary sinus itself may be related to the size of the sinus ${ }^{(15)}$. Accordingly, we demonstrated that maxillary sinus wall thickness is an indicator of poor surgical outcome.

The success rate of surgical outcomes for CRS patients across surgeons varies. The experience and technique of the surgeon are important factors related to the successfulness of treatment. Surgical studies contain congenital bias, that is, procedures or interventions are not executed in a uniform way; there is also a lack of patient-blinding to the surgical intervention and performance bias, which is also the case between different surgeons. Therefore, it is crucial to establish a standard surgical protocol in order to avoid the impact of the confounding effect of surgical techniques on patient outcomes. Therefore, over the past 10 years, we have made an effort to standardize surgical procedures in order to eliminate differences among surgeons. The standard 8-step FESS procedure is based on Stammberger and Kennedy's methods, which sequentially remove the obstruction of the drainage pathway anterior to posterior to reach the sphenoid sinus ostium; then, it is moved from posterior back to anterior along the identified skull base until the AEA is identified and the frontal sinus is opened. If the diseased sinuses are limited, the surgical procedure can be tailored so that the normal sinus mucosa and draining pathway are exposed and identified; this is also a way to educate beginners to understand FESS in an organized method.

In 1992, it was first suggested that chronic inflammation of the bony framework of paranasal sinuses plays a pivot role in the pathophysiology of CRS; this hypothesis was further confirmed by subsequent animal studies ${ }^{(16)}$. Geogalas et al. proposed a global osteitis scoring scale as an indicator of revised sinus surgery ${ }^{(17)}$. Snidvongs et al. proposed that the osteitic sinus bone is a surrogate of tissue or serum eosinophilia in CRS patients ${ }^{(18)}$. Osteitis changes in the sinus bone are present in heterogeneous, irregular bone in areas of growth and destruction. Some studies have
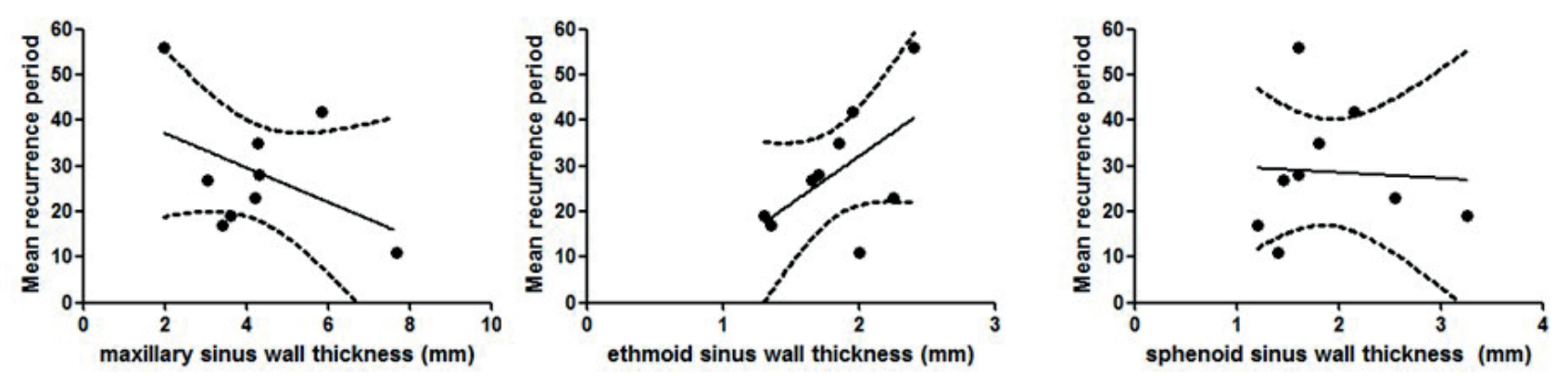

Figure 4. Mean recurrence period showed no significant relationship compared to the maxillary, ethmoid, and sphenoid sinus wall thickness (linear regression; $p>0.05)$. 


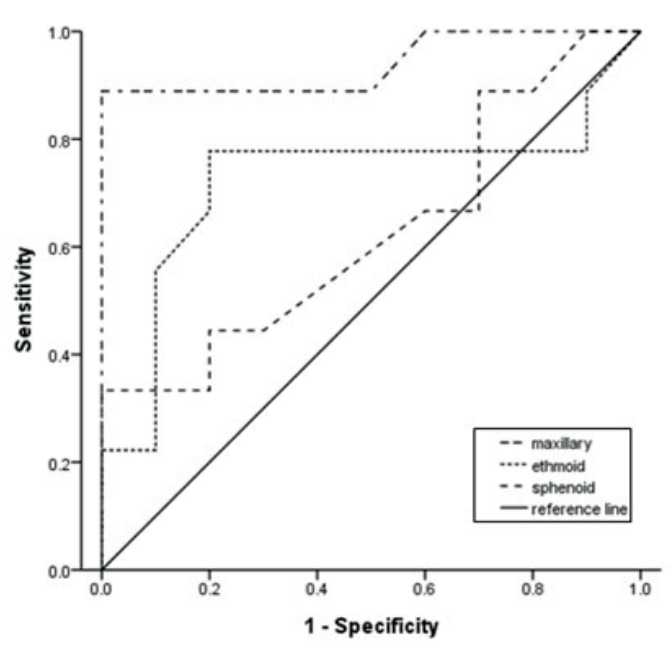

ROC curve

Figure 5. ROC curve shows the cut-off values for best sensitivity and specificity of each sinus:3.03mm, $1.63 \mathrm{~mm}$, and $1.75 \mathrm{~mm}$ in maxillary, ethmoid, and sphenoid sinus, respectively. The ROC curve of maxillary sinus has maximal AUC (area-under-curve) of 0.94 .

proposed that bone thickness can predict the severity of osteitis ${ }^{(19,20)}$. Recently, sinus osteitis and subsequent bony remodeling were also suggested as a contributing factor to refractory CRS. At a microscopic level, osteitis is associated with eosinophilic inflammation and may represent a method to predict patients with P-gp overexpression by using an epithelial-to-background staining ratio; increased osteitis burden is associated with increased P-gp membranous expression in CRS ${ }^{(21-23)}$. In our study, the sinus bony walls in group $A$ were significantly thicker than those in group $C$ and $D$; in group $B$, only the maxillary sinus wall was significantly thicker than that of the control group. These results reflect the importance of the role of a surgeon in evaluating surgical outcomes. Our data also suggest that a postero-lateral maxillary sinus wall thickness of $3.03 \mathrm{~mm}$ should be the cut-off value in order to predict refractory CRS.

The existence of bacterial biofilms (BBF) has also been proposed to be associated with osteitis in CRS ${ }^{(10)}$. Biofilm formation might reflect the severity, chronicity, or, both, of sinus infection; therefore, the release of inflammatory mediators would stimulate osteoblast activity, inducing bony remodeling and osteitis. Osteitis may further spread the pathogen either via the haversian canal system hematogenously or from direct local invasion ${ }^{(24)}$. Intraepithelial bacteria are also found in CRS patients ${ }^{(25)}$. Considering the histology of sinus mucosa, these factors represent the depth of involvement of inflammatory process and indicate a prolonged treatment course. Although it did not reach statistical significance, maxillary sinus wall thickness is also related to shortened time to recurrence (Figures 3, 4); therefore, compared to $L-M$ scores of sinus $C T$, sinus wall thickness may represent greater proximity for the chronicity of CRS. At present, the mainstay of sinus surgery still focuses on restoring ventilation, yet no specific surgical method has proved to be effective in treating osteitis associated with CRS. Instead, long-term or topical antibiotic treatment is administrated in cases of sinus osteitis and refractory sinsusitis ${ }^{(26)}$.

It has previously been reported that eosinophilic inflammation in the sinonasal tissues is correlated with the advanced severity of CRS and the poor outcomes associated with FESS ${ }^{(12)}$. Recent evidence has shown that eosinophilic inflammation in Caucasians CRS patients with polyps does not affect Asian to the same extent. A study from Thailand indicated a time-shifting migration of neutrophilic inflammation to eosinophilic inflammation, and a study from Korea suggested that eosinophilic inflammation may not be related to surgical outcome in Korean CRS patients ${ }^{(27,28)}$. In our study, increased peripheral eosinophil numbers had a limited impact on surgical outcomes, suggesting that the clinical implication of eosinophilic inflammation might be different in Asian patients. Nevertheless, our study strengthens the hypothesis that increased eosinophil numbers are a poor indicator of CRS outcomes. Blood eosinophilia is induced from proliferation of eosinophil progenitor from bone marrow (myeloproliferative) or clonal expansion of peripheral eosinophil in the blood stream ${ }^{(29,30)}$. The proposal that refractory CRS represents a local manifestation of systemic inflammatory disease is supported by our results. Our study suggested that, for those patients with obvious sinus wall thickening, more detailed preoperative consultation and laboratory tests and more aggressive postoperative medical treatment are needed.

There are several limitations of this study. First, we had small case numbers. Second, there are individual differences in the tolerance of sinusitis, as revision surgery was used as the judgment for refractory sinusitis. Despite the limitations, the knowledge gained in our study provides crucial information to guide surgical selection in CRS patients.

\section{Conclusion}

In summary, a variety of factors lead to refractory CRS. As a result, treatment for refractory CRS is a great challenge for ENT surgeons. Thickness of postero-lateral maxillary sinus wall of than $3.03 \mathrm{~mm}$ indicates possibility for revised surgery. For those CRS patients with thickened sinus wall in which we expect poor outcomes, further research is needed in order to justify the surgical procedure in such a probable systemic inflammatory disease.

\section{Acknowledgement}

This study was supported by a grant (100-2314-B-002-044-) from The Ministry of Science and Technology (MOST) of the Republic of China. 


\section{Authorship contribution}

YTH, LKY, CPY defined the research theme. LKY and CPY analyzed the data. YTH interpreted the results and wrote the paper. All authors discussed analyses, interpretation, and presentation. All authors have contributed to, seen and approved the manus- cript.

\section{Conflict of interest}

There are no financial or other relationships that could lead to a conflict of interest.

\section{References}

1. Jankowski R. Revisiting human nose anatomy: phylogenic and ontogenic perspectives. Laryngoscope 2011; 121: 2461-2467.

2. Fokkens WJ, Lund VJ, Mullol J, et al. European position paper on rhinosinusitis and nasal polyps 2012. Rhinol. 2012; Suppl 23:1-298.

3. Rosenfeld RM, Andes D, Bhattacharyya N, et al. Clinical practice guideline: adult sinusitis. Otolaryngol Head Neck Surg. 2007; 137(3 Suppl):S1-31.

4. Ragab SM, Lund VJ, Scadding G. Evaluation of the medical and surgical treatment of chronic rhinosinusitis: a prospective, randomised, controlled trial. Laryngoscope 2004; 114: 923-930.

5. Georgalas C, Cornet M, Adriaensen G, et al. Evidence-based surgery for chronic rhinosinusitis with and without nasal polyps. Curr Allergy Asthma Rep. 2014 ; 14:427.

6. Desrosiers M. Refractory chronic rhinosinusitis: pathophysiology and management of chronic rhinosinusitis persisting after endoscopic sinus surgery. Curr Allergy Asthma Rep. 2004; 4: 200-207.

7. Lund VJ, Mackay IS. Staging in rhinosinusitus. Rhinology 1993; 31: 183-184.

8. Hamilos DL. Host-microbial interactions in patients with chronic rhinosinusitis. J Allergy Clin Immunol. 2014 ; 133: 640-653.

9. Smith TL, Mendolia-Loffredo S, Loehrl TA, et al. Predictive factors and outcomes in endoscopic sinus surgery for chronic rhinosinusitis. Laryngoscope 2005; 115: 2199-2205.

10. Dong D, Yulin Z, Xiao W, et al. Correlation between bacterial biofilms and osteitis in patients with chronic rhinosinusitis. Laryngoscope 2014 ; 124: 1071-1077.

11. Robinson JL, Griest $S$, James KE, Smith TL. Impact of aspirin intolerance on outcomes of sinus surgery. Laryngoscope 2007; 117 : 825-830.

12. Zadeh MH, Banthia V, Anand VK, Huang C. Significance of eosinophilia in chronic rhinosinusitis. Am J Rhinol. 2002; 16: 313-317.

13. Yeh TH, Hsu WC, Chen YS, Hsu CJ, Lee SY. Decreased connexin 43 expression correlated with eosinophil infiltration in nasal polyps. Am J Rhinol. 2005 ;19:59-64.

14. Zollikofer CP, Weissmann JD. A morphogenetic model of cranial pneumatization based on the invasive tissue hypothesis. Anat Rec (Hoboken). 2008 ; 291: 1446-1454.

15. Cho SH, Shin KS, Lee YS, et al. Impact of chronic rhinosinusitis and endoscopic sinus surgery on bone remodeling of the paranasal sinuses. Am J Rhinol. 2008 ; 22: 537-541.

16. Norlander T, Forsgren K, Kumlien J, Stierna P, Carlsoo B. Cellular regeneration and recovery of the maxillary sinus mucosa. An experimental study in rabbits. Acta Otolaryngol Suppl. 1992; 492: 33-37.

17. Georgalas C, Videler W, Freling N, Fokkens W. Global Osteitis Scoring Scale and chronic rhinosinusitis: a marker of revision surgery. Clin Otolaryngol. 2010; 35: 455-461.

18. Snidvongs K, McLachlan R, Chin D, et al. Osteitic bone: a surrogate marker of eosinophilia in chronic rhinosinusitis. Rhinology 2012; 50: 299-305.

19. Lee JT, Kennedy DW, Palmer JN, Feldman M, Chiu AG. The incidence of concurrent osteitis in patients with chronic rhinosinusitis: a clinicopathological study. Am J Rhinol. 2006; 20: 278-282.

20. Kim HY, Dhong $\mathrm{HJ}$, Lee $\mathrm{HJ}$, et al, Hyperostosis may affect prognosis after primary endoscopic sinus surgery for chronic rhinosinusitis. Otolaryngol Head Neck Surg. 2006; 135: 94-99.

21. Feldman RE, Lam AC, Sadow PM, et al. P-glycoprotein is a marker of tissue eosinophilia and radiographic inflammation in chronic rhinosinusitis without nasal polyps. Int Forum Allergy Rhinol. 2013; 3: 684-687.

22. Bleier BS, Nocera $A L$, lqbal $H$, et al. P-glycoprotein functions as an immunomodulator in healthy human primary nasal epithelial cells. Int Forum Allergy Rhinol. 2013; 3: 433-438.

23. Günel C, Feldman RE, Bleier BS. Osteitis is associated with P-glycoprotein overexpression in patients with chronic sinusitis without nasal polyps. Am J Rhinol Allergy 2014; 28: 99-102.

24. Perloff JR, Gannon FH, Bolger WE, Montone KT, Orlandi R, Kennedy DW. Bone involvement in sinusitis: an apparent pathway for the spread of disease. Laryngoscope 2000; 110: 2095-2099.

25. Plouin-Gaudon I, Clement S, Huggler E, et al. Intracellular residency is frequently associated with recurrent Staphylococcus aureus rhinosinusitis. Rhinology $2006 ; 44$ : 249-254.

26. Ezzat WF, Fawaz SA, Rabie H, Hamdy TA, Shokry YA. Effect of topical ofloxacin on bacterial biofilms in refractory postsinus surgery rhino-sinusitis. Eur Arch Otorhinolaryngol. 2015 Sep;272(9):2355-61.

27. Katotomichelakis M, Tantilipikorn $P_{\text {, }}$ Holtappels G, et al. Inflammatory patterns in upper airway disease in the same geographical area may change over time. Am J Rhinol Allergy. 2013; 27: 354-360.

28. Kim SY, Park JH, Rhee CS, Chung JH, Kim JW. Does eosinophilic inflammation affect the outcome of endoscopic sinus surgery in chronic rhinosinusitis in Koreans? Am J Rhinol Allergy. 2013; 27: e166-169.

29. Simon HU, Rothenberg ME, Bochner BS, et al. Refining the definition of hypereosinophilic syndrome. J Allergy Clin Immunol. 2010; 126: 45-49.

30. Valent $P$, Klion AD, Horny HP, et al. Contemporary consensus proposal on criteria and classification of eosinophilic disorders and related syndromes. J Allergy Clin Immunol. 2012; 130: 607-612.

Te-Huei Yeh MD, PhD

Department of Otolaryngology

National Taiwan University Hospital

No 7, Chung-Shan south road

Taipei 100

Taiwan

Tel: +886-2-23123456 ext.65223

Fax: +886-2-23410905

E-mail: tehueiyeh@ntu.edu.tw 